\title{
ACERCA DEL CARÁCTER CUANTIFICADOR DE LAS LOCUCIONES ADVERBIALES CON LA PREPOSICIÓN 'HASTA'
}

\author{
Monika Lisowska \\ Universidad de Szczecin \\ http://dx.doi.org/10.18778/8220-201-4.13
}

\section{Resumen}

El objetivo del presente artículo es describir las propiedades cuantificacionales de las locuciones adverbiales con la preposición 'hasta'. Basándose sobre todo en el trabajo de Lamíquiz (1991), la autora identifica los modos lógicos de la sustancia cuantificada por estas locuciones, el modelo de referencia cuantificadora adoptado y, finalmente, los tipos de cuantificación realizada, distinguidos según tres criterios diferentes.

Palabras clave: Semántica, sintaxis, cuantificación lingüística, locución adverbial.

\section{1.}

\section{Introducción}

La clase de adverbios propiamente dichos es un grupo de palabras cuyos elementos integrantes, de acuerdo con su significado, suelen dividirse tradicionalmente en las siguientes clases: de lugar, de tiempo, de modo, de cantidad, de afirmación, de negación, de duda ( $R A E-A S A L E, 2009$ : $\$ 30.2 e$ ). Además, la lengua cuenta también con ciertas agrupaciones fijas de palabras con carácter 
adverbial ${ }^{1}$, de las cuales muchas vienen a suplir la ausencia de adverbios que indiquen ciertas nociones concretas (Álvarez Martínez, 2000: 16).

Las agrupaciones a las que nos referimos reciben diferentes denominaciones en los estudios dedicados al tema: expresiones, giros o, con mayor frecuencia, locuciones adverbiales. Una de sus características destacadas, referentes a su forma, es la estructura de un grupo preposicional en la mayoría de los casos (RAE-ASA$L E, 2009: \$ 30.15 b)$.

En el presente artículo, la clase de las locuciones adverbiales que nos ocupa presenta precisamente la estructura de este tipo. Se trata de aquellas con la preposición 'hasta', donde dicha preposición funciona como enlace de un grupo preposicional. Los demás elementos desempeñan el papel de término de sintagma, $\mathrm{y}$, habitualmente, se presentan bajo la forma de sintagmas nominales, p. ej.: hasta el cuello, hasta el moño, hasta la médula, hasta las cachas, hasta los topes. Se dan también casos aislados de términos en forma de oraciones con verbo en infinitivo (hasta decir basta, hasta más no poder) o sintagmas adverbiales (hasta no más, hasta $\underline{\text { arriba). }}$. Opinamos que todas estas locuciones comunican cierto tipo de cuantificación, cuyas características vamos a presentar más adelante.

Aunque en el presente trabajo clasificamos todas las locuciones en cuestión como adverbiales, téngase en cuenta que una parte de ellas, debido al cambio semántico, es clasificada por los lexicólogos como locuciones de otra categoría gramatical. Se trata, ante todo, de aquellas que solían utilizarse como ponderativas modificando el adjetivo 'harto', el cual quedó eliminado de estas expresiones. La unidad resultante de la elipsis del adjetivo adquiere el valor semántico y categorial de todo el grupo adjetival, de ahí que las locuciones como hasta el moño (CLAVE, s.v. 'moño') o hasta el coco (DELE, s.v. 'coco') queden registradas como locuciones

1 El carácter adverbial de una unidad léxica está relacionado, sobre todo, con la posibilidad de actuar como modificador de grupos verbales, adjetivales y adverbiales (RAE-ASALE, 2009: \$30.1a). 
adjetivales con el sentido de 'muy harto'2 . La misma suerte han corrido las locuciones hasta la bandera (CLAVE, DLE, s.v. 'bandera') y hasta los topes (DELE, MOLINER, s.v. 'tope'), clasificadas como adjetivales por la elipsis del adjetivo 'lleno'.

\section{2. \\ Cuantificación lingüística: características generales}

No cabe duda de que la cuantificación en el discurso va más allá de "expresar numéricamente una magnitud de algo" (DLE, s.v. 'cuantificar', 1. a acepción), puesto que las ciencias humanas, y entre ellas la lingüística, abarcan también lo cualitativo ${ }^{3}$ (Lamíquiz 1991: 18).

En lo que concierne a este fenómeno, vamos a recurrir sobre todo al trabajo de Lamíquiz (1991), siendo este un estudio muy detallado sobre diferentes cuestiones relacionadas con la cuantificación lingüística. Así, siguiendo al mencionado lingüista, en primer lugar, cabe subrayar que no son las unidades lexemáticas las que permiten verse valoradas cuantitativamente, sino las sustancias extralingüísticas, sobre todo la conceptual, que se formalizan en estas unidades (Lamíquiz, 1991: 23).

Sin embargo, hay que recordar que no toda sustancia conceptual es susceptible de someterse a cuantificación. Respecto a ello Lamíquiz (1991: 34) advierte que “(...) dado que únicamente se

2 Algunos autores siguen considerando estas locuciones como adverbiales, advirtiendo que dejan de serlo con el verbo 'estar' (García Page, 2007: 129).

3 De acuerdo con esta observación, la RAE y la ASALE presentan la acepción 'no técnica' del término cuantificación, entendida como la expresión de la medida de algo que puede realizarse tanto numéricamente como mediante otras formas de estimación, como, por ejemplo, los cuantificadores indefinidos (RAE-ASALE, 2009: $\$ 19.1 \mathrm{a})$. 
puede cuantificar lo graduable, se da restricción lógica de cuantificación con las unidades lexemáticas que supongan semánticamente lo total o lo nulo 4 ." A continuación, el autor indica diferentes formas de existencia de la sustancia conceptual, llamadas modos lógicos, que influyen en el valor significativo de una unidad lexemática: los conceptos, los ejemplares, las clases, los atributos y las acciones (Lamíquiz, 1991: 34). Según informa acertadamente, excepto el modo lógico concepto, “(...) todos los demás modos de sustancia son mesurables y, en consecuencia, admiten su cuantificación (...)” (Lamíquiz, 1991: 37).

A todo lo anterior cabe añadir que cada tipo de cuantificación necesita de un operador o cuantificador ${ }^{5}$, cuya tarea consiste en expresar el cómputo, la medición o la evaluación sobre la noción cuantificada (RAE-ASALE, 2009: \$19.1c). Hasta el día de hoy, los lingüistas han propuesto diferentes clasificaciones de cuantificadores, establecidas en función de diversos criterios. Uno de ellos es la clase sintáctica a la que pertenecen las palabras o locuciones cuantificadoras, distinguiéndose según ese criterio los cuantificadores adjetivales, pronominales y adverbiales ${ }^{6}$ (RAE-ASALE, 2009: $\$ 19.2 a)$. De acuerdo con esta clasificación, las locuciones adverbiales con 'hasta' pertenecerán, obviamente, a la clase de los cuantificadores adverbiales.

4 Por ejemplo: ${ }^{\star}$ Está poco vacío; ${ }^{*}$ Iba muy descalza (Lamíquiz, 1991: 33).

5 Recordamos que la noción de cuantificador en lingüística hace referencia a una clase semántica de palabras y no a una nueva categoría léxica (Leonetti, 2007: 11).

6 Según observa Leonetti (2007: 81), son básicamente los adverbios y los determinantes los que más presencia tienen en el grupo de cuantificadores. 


\section{Las locuciones adverbiales con hasta: característica cuantificacional}

\section{1.}

\section{Módos lógicos de la sustancia conceptual cuantificada}

Aunque no se puede hablar de una correspondencia rigurosa entre modos lógicos y categorías sintácticas (Lamíquiz, 1991: 43), es cierto que algunas categorías suelen ofrecer siempre el mismo modo lógico de la sustancia conceptual, como, por ejemplo, los verbos la presentan como una acción, y los adjetivos como un atributo.

Según es de suponer, la pertenencia a una determinada clase sintáctica delimita el poder de cada cuantificador, pudiendo este modificar directamente solo aquellas unidades lexemáticas cuya clase gramatical es sintácticamente compatible con la suya. Por ello, para descubrir cuál es el modo lógico de la sustancia conceptual modificada por las locuciones adverbiales con 'hasta', es necesario analizar el comportamiento sintáctico de estas.

Así, en primer lugar, las locuciones que nos interesan aparecen como complementos de verbos. Cabe advertir que en muchos casos se trata de modificar verbos concretos, los cuales junto con las locuciones en cuestión forman colocaciones ${ }^{7}$, por ejemplo: (meterse) hasta los codos; (meter la pata) hasta el fondo / hasta las orejas; (armarse) hasta los dientes; (echar/vomitar) hasta la (primera) papilla; (llenar) hasta los topes / hasta arriba / hasta la bandera; (endeudarse) hasta el cuello / hasta las cejas; (calar, empapar) hasta los huesos.

No son muy frecuentes las locuciones con 'hasta' que no guarden tanta solidaridad con determinados verbos $y$, por consiguiente, puedan relacionarse con diferentes acciones o actividades. He aquí el caso de hasta decir basta, que se muestra particularmente flexible en este aspecto: "No es que me disguste absolutamente

7 Es decir, sintagmas que “(...) presentan cierto grado de restricción combinatoria determinada por el uso” (Corpas Pastor, 1996: 53). Las colocaciones del tipo verbo más locución verbal han sido denominadas colocaciones complejas (Koike, 2001: 55-60). 
todo lo que hoy escuchamos hasta decir basta en las radios (...)"; "Sí, le dieron cera a sus tobillos hasta decir basta (...)"; "(...) se ayudan hasta decir basta (...)”; “(...) lo radiografiaron hasta decir basta (...)" (CREA).

Aparte de ser modificadores de verbos en forma personal, es habitual que las locuciones en cuestión modifiquen el significado de participios. Considérense los siguientes ejemplos: “(...) un maorí tatuado hasta las cejas (...)”; “(...) hundido hasta el cuello en el fango de la corrupción.”; “(...) conmovido hasta los tuétanos (...)"; "Enamorado hasta las trancas (...)"; “(...) presionado hasta las cachas por los Estados Unidos (...)" (CREA).

Una gran parte de las locuciones con la preposición 'hasta' pueden funcionar, además, como adyacentes de adjetivos. A este respecto, en primer lugar, se observa cierta restricción de su uso debida a la ya mencionada afinidad de algunas de estas locuciones con el adjetivo 'harto', hoy en día ausente y dado por sobrentendido en las expresiones con los verbos 'estar' o 'tener'. A este grupo pertenecen, entre otros: ('estar [harto] / tener a alguien [harto]') hasta el coco, hasta el cogote, hasta el gollete, hasta el gorro, hasta el moño, hasta la cocorota, hasta la coronilla, hasta las narices, hasta los pelos. Merece observar que algunas de esas locuciones son capaces de romper la restricción de uso y pasar a modificar participios: "Ahora imagine que está endeudado hasta el cogote"; "Ex ministro Bermejo metido hasta las narices (...)" (CREA).

En cambio, las locuciones con la preposición 'hasta' capaces de modificar otros adjetivos, distintos a 'harto', son menos numerosas: “(...) susceptibles hasta decir basta (...)”; “(...) debo serte franco hasta el fondo (...)"; "James Bond era machista hasta la médula (...)"; "Cocainómana hasta las cachas (...)"; (...) repleto hasta las trancas de rollos de papel higiénico (...)” (CREA).

Según lo acabamos de presentar, las únicas funciones sintácticas de las locuciones adverbiales aquí debatidas son la de complemento circunstancial o aditamento y la de adyacente de un adjetivo, con lo cual cabe concluir que la sustancia conceptual cuantificada por las locuciones con 'hasta' se presenta únicamente bajo los dos siguientes modos lógicos: el de acción (verbos) y el de atributo (participios, adjetivos). 


\section{2.}

\section{Tipo de modelo de referencia cuantificadora}

Al querer producir una matización cuantificadora, el hablante hace uso de las referencias de mesurabilidad, utilizando al mismo tiempo algún modelo de referencia que le sirve de base. En este aspecto, Lamíquiz (1991: 47-48) subraya la relatividad de toda cuantificación debido a que esta implica “(...) una comparación de la sustancia que se quiere ponderar con una medida modelo previamente instaurada como unidad de referencia cuantificadora". El mismo autor señala que en la lengua existen tres tipos de baremos relativos de referencia medidora (Lamíquiz, 1991: 48-50):

a) la unidad singular, que se caracteriza por el uso de los numerales;

b) el modelo umbral de lo normal, que parte de una cantidad modelo percibida como umbral de lo normal y está basado en los conocimientos pragmáticos de los interlocutores situados en el mismo contexto o espacio sociocultural;

c) otra sustancia significativamente comparable o la misma sustancia en otro objeto, que aparece de manera explícita en el enunciado que expresa algún tipo de comparación.

En el caso de las locuciones objeto de nuestro estudio, la presencia de la preposición hasta impone que el significado literal de cada una de ellas sea el de señalar un límite, que es espacial en la mayoría de los casos, y se comunica en la segunda parte del sintagma: el término.

Admitiendo que el límite, ese grado máximo al que es posible llegar, en este tipo de locuciones expresa metafóricamente 'gran cantidad' o 'mucha intensidad'; es lógico suponer que este quede fijado respecto a un punto neutro, considerado como común o habitual. Por todo ello, parece legítimo afirmar que el tipo de modelo de referencia cuantificadora adoptado en la cuantificación realizada por las locuciones adverbiales con 'hasta' es el anteriormente comentado modelo umbral de lo normal. 


\section{3.}

\section{Tipo de cuantificación}

El empleo de un determinado modelo de referencia cuantificadora señala necesariamente el tipo de cuantificación lingüística que se está llevando a cabo. A este respecto, Lamíquiz (1991: 51) indica los dos siguientes tipos generales:

a) la cuantificación unitaria, es decir, la que en el texto enunciativo presenta una sola sustancia de la que se informa sobre su cantidad;

b) la cuantificación comparativa, es decir, la que opera con dos elementos sustanciales, cuya cantidad es el objeto de la comparación.

Según es de esperar, el segundo tipo de cuantificación emplea siempre el tercer modelo de unidad de medición cuantificadora, o sea, otra sustancia comparable. Mientras tanto la cuantificación unitaria puede emplear como unidad de referencia la unidad matemática o el umbral de lo normal (Lamíquiz, 1991: 51).

Aplicando esta distinción a la cuantificación realizada por las locuciones adverbiales con 'hasta', no cabe duda de que estamos ante la cuantificación unitaria, dado que, según lo hemos comentado supra, este tipo de locuciones, al actuar como operadores, adoptan el modelo umbral de lo normal como modelo de referencia cuantificadora.

Ahora bien, el uso del determinado modelo de referencia cuantificadora no es el único criterio que se puede adoptar para establecer una tipología de la cuantificación lingüística. También se pueden hacer, al menos, dos distinciones siguientes 8 :

a) la sintáctica, en función de la clase sintáctica a la que corresponden o se asimilan las unidades cuantificadoras: cuantificación adjetival, pronominal, adverbial;

b) la semántica, en función de las nociones cuantificadas: cuantificación de individuos, de materia o sustancia, de grado.

Respecto a la clasificación sintáctica, según ha quedado plasmado en las líneas anteriores, las locuciones adverbiales con

8 Distinción nuestra, hecha a base de las clasificaciones de cuantificadores presentadas en la última gramática académica (RAE-ASA$L E, 2009: \$ 19.2 . a)$. 
'hasta' pertenecen al grupo de los cuantificadores adverbiales, con lo cual es lógico que la cuantificación que realizan sea también de este tipo, es decir, adverbial.

En cambio, tomando en cuenta la distinción semántica de la cuantificación, estamos sin duda ante la cuantificación de grado, es decir aquella en la que los operadores no ligan “(...) variables relacionadas con los individuos u objetos que están dentro de su alcance, ya que la variable es de otra naturaleza" (Muñoz Núñez, 2010: 463). En este tipo de cuantificación, las variables denotan “(...) niveles jerárquicamente ordenados en una escala” (Sánchez López, 1999: 1090), es decir, expresan el grado en que se manifiesta una propiedad.

Efectivamente, las locuciones adverbiales con 'hasta', modificando sustancias conceptuales presentadas bajo el modo lógico de acción y el de atributo, se especializan en destacar que lo denotado se encuentra en su grado más alto o muy cerca de él. Sirvan como ejemplos los siguientes enunciados: Corrupto hasta las cachas, pero muy profesional, sí. ("Punta Escarlata”, cap. 8, 0:15:29); Sí, detuvimos a un camello que iba puesto hasta las cejas. ("Estoy vivo", cap. 5, 0:36:52); Porque íbamos de farol y usted se lo tragó hasta las trancas. ("Ministerio del tiempo", cap. 33, 0:56:16).

Según se puede observar, dependiendo del tipo de la sustancia conceptual modificada (y también del propósito del hablante), las locuciones con 'hasta' al referirse literalmente a un límite, metafóricamente llegan a significar 'en un grado máximo', 'intensamente', e incluso 'por completo/totalmente’. Estos valores semánticos las sitúan muy arriba en la escala de cuantificación de grado.

Merece añadir que pueden darse casos de lectura no cuantificativa, o sea, de interpretación literal de algunas de estas locuciones, impuesta por el significado del verbo modificado, resultando aquel compatible con el significado literal de una locución concreta, como, por ejemplo: abrocharse (la camisa) hasta el cuello (sentido literal, sin cuantificación); endeudarse hasta el cuello (sentido metafórico, expresión de cuantificación). Seguramente, en el caso de activarse el significado literal ya no estamos ante locuciones adverbiales, sino ante sintagmas preposicionales no idiomáticos, donde la unión entre el enlace y el término no es fija. 


\section{Consideraciones finales}

Admitiendo que la cuantificación en lingüística abarca no solo lo cuantitativo, sino también lo cualitativo, no cabe duda de que las locuciones con 'hasta' funcionan como cuantificadores.

El carácter adverbial de estas locuciones, reflejado en el hecho de cuantificar únicamente la sustancia conceptual presentada bajo el modo lógico de acción o de atributo, hace que, conforme al criterio sintáctico, la cuantificación realizada por estas locuciones sea también adverbial.

En cambio, gracias a sus propiedades semánticas, las locuciones con 'hasta', al intensificar lo denotado por adjetivos o verbos (en numerosas colocaciones en el caso de esos últimos), realizan la cuantificación de grado y la sitúan en una posición muy alta en la escala prevista para esta categoría semántica, con lo cual estamos ante una cuantificación de grado positivo. Estas propiedades semánticas se deben al modelo de referencia cuantificadora adoptado (modelo umbral de lo normal), el cual, por su parte, es propio de la cuantificación unitaria.

Finalmente, conviene subrayar que el valor cuantificador de las locuciones adverbiales con 'hasta', realizado gracias a su uso metafórico, puede desaparecer en ciertas circunstancias semánticas, originándose en esos casos la interpretación literal del significado de todo el sintagma preposicional.

\section{Referencias bibliográficas}

Álvarez Martínez, Ma Á. (2000), El adverbio, Madrid: Arco Libros.

Corpas Pastor, G. (1996), Manual de fraseología española, Madrid: Gredos.

García Page, M. (2007), "Esquemas sintácticos de formación de locuciones adverbiales", Moenia, 13, 121-144.

Koike, K. (2001), Colocaciones léxicas en el espanol actual estudio formal y léxico semántico, Alcalá de Henares: Universidad de Alcalá / Takushoku University. 
Lamíquiz, V. (1991), La cuantificación lingüística y los cuantificadores, Madrid: UNED.

Leonetti, M. (2007), Los cuantificadores, Madrid: Arco Libros.

Muñoz Núñez, Ma D. (2010), "Algunos aspectos de la cuantificación de grado (con especial atención a la llamada 'cuantificación de grado verbal'”, Nueva Revista de Filología Hispánica, Vol. 58, núm. 2, 461-482.

(RAE-ASALE) Real Academia Española y Asociación de Academias de la Lengua Española (2009), Nueva gramática de la lengua española, Madrid: Espasa.

Sánchez López, C. (1999), "Los cuantificadores: clases de cuantificadores y estructuras cuantificativas", en I. Bosque y V. Demonte (coords.), Gramática descriptiva de la lengua española, Vol. 1, Madrid: Espasa Calpe, 1025-1128.

\section{Diccionarios}

(CLAVE) Diccionario Clave, [en línea] <http://clave.smdiccionarios.com $>$, [fecha de consulta: 30.05 .2018 ].

(DELE) Martínez López, J. A. \& Myre Jørgensen, A. (2009), Diccionario de expresiones y locuciones del español, Madrid: Ediciones de la Torre.

(DLE) Diccionario de la Lengua Española, [en línea] <http://dle. rae.es>, [fecha de consulta: 30.05.2018].

(MOLINER) Diccionario de uso del español, por María Moliner, edición electrónica, Madrid: Editorial Gredos (2008).

\section{Fuentes de datos}

Real Academia Española: Banco de datos (CREA). Corpus de referencia del español actual, [en línea] <http://www.rae.es $>$, [fecha de consulta: 22.05.2018].

Estoy vivo, serie de televisión, [en línea] <http://www.rtve.es/television/series-tve/>, [fecha de consulta: 12.06.2018].

Ministerio del Tiempo, serie de televisión, [en línea] <http://www. rtve.es/television/series-tve/>, [fecha de consulta: 15.06.2018].

Punta Escarlata, serie de televisión, [en línea] <http:mitele.es $>$, [fecha de consulta: 24.06.2018]. 